\title{
Front Matter: Volume 9289
}

, "Front Matter: Volume 9289," Proc. SPIE 9289, 12th Education and Training in Optics and Photonics Conference, 928901 (3 February 2015); doi:

$10.1117 / 12.2076221$

SPIE Event: 12th Education and Training in Optics and Photonics Conference, 2013, Porto, Portugal 


\title{
PROCEEDINGS OF SPIE
}

\section{2th Education and Training in Optics and Photonics Conference}

\author{
Manuel F. P. C. Martins Costa \\ Mourad Zghal \\ Editors
}

23-26 July 2013

Porto, Portugal

Sponsored by

ICO_-International Commission for Optics

IEEE-The Photonics Society

The Optical Society

SPIE

Published by

SPIE 
The papers included in this volume were part of the technical conference cited on the cover and title page. Papers were selected and subject to review by the editors and conference program committee. Some conference presentations may not be available for publication. The papers published in these proceedings reflect the work and thoughts of the authors and are published herein as submitted. The publisher is not responsible for the validity of the information or for any outcomes resulting from reliance thereon.

Please use the following format to cite material from this book:

Author(s), "Title of Paper," in 12th Education and Training in Optics and Photonics Conference, edited by Manuel F. P. C. Martins Costa and Mourad Zghal, Proceedings of SPIE Vol. 9289 (SPIE, Bellingham, WA, 2014) Article CID Number.

ISSN: 0277-786X

ISBN: 9781628413649

Published by

SPIE

P.O. Box 10, Bellingham, Washington 98227-0010 USA

Telephone +1 3606763290 (Pacific Time) · Fax +1 3606471445

SPIE.org

Copyright @ 2014, SPIE, IEEE, OSA, and ICO

Printed in the United States of America.

Publication of record for individual papers is online in the SPIE Digital Library.

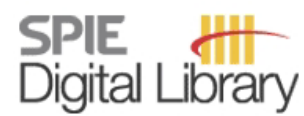

SPIEDigitalLibrary.org

Paper Numbering: Proceedings of SPIE follow an e-First publication model, with papers published first online and then in print and on CD-ROM. Papers are published as they are submitted and meet publication criteria. A unique, consistent, permanent citation identifier (CID) number is assigned to each article at the time of the first publication. Utilization of CIDs allows articles to be fully citable as soon as they are published online, and connects the same identifier to all online, print, and electronic versions of the publication. SPIE uses a six-digit CID article numbering system in which:

- The first four digits correspond to the SPIE volume number.

- The last two digits indicate publication order within the volume using a Base 36 numbering

system employing both numerals and letters. These two-number sets start with 00, 01, 02, 03, 04, $05,06,07,08,09,0 A, 0 B \ldots 0 Z$, followed by 10-1Z, 20-2Z, etc.

The CID Number appears on each page of the manuscript. The complete citation is used on the first page, and an abbreviated version on subsequent pages. Numbers in the index correspond to the last two digits of the six-digit CID Number. 


\title{
Contents
}

\author{
ix Authors \\ xiii Conference Committee
}

\section{SESSION 1 GENERAL CONCEPTS}

928902 A correlation of thin lens approximation to thick lens design by using context based method in optics education [9289-1]

928903 m-Learning and holography: Compatible techniques? [9289-2]

928904 Developing critical thinking, creativity and innovation skills of undergraduate students (Invited Paper) [9289-3]

928905 CApability Matrix for Photonics Up-Skilling (CAMPUS) [9289-4]

928906 What can we teach using adaptive optics? [9289-5]

928907 Inspiring future experimental scientists through questions related to colour [9289-6]

928908 Teaching quantum mechanics with the Hong-Ou-Mandel interferometer [9289-7]

928909 Astronomical phenomena: events with high impact factor in teaching optics and photonics [9289-8]

9289 OA The Puerto Rico Photonics Institute [9289-9]

9289 OB Concept of the International Project University: learning without borders [9289-10]

9289 OC Formulation of didactic interest of the laws of refraction of light [9289-11]

9289 OD Diffraction operators in paraxial approach [9289-63]

$9289 \mathrm{OE}$ Top lateral refraction and reflection of polarized light in lenses, coplanar lens system, applications [9289-64]

9289 OF Teaching optical dimensional metrology of surfaces and international standards [9289-67]

9289 OG Using ray matrices to derive analytical expressions of optical aberrations [9289-86]

$9289 \mathrm{OH}$ Single-photon interference experiment for high schools [9289-91]

9289 Ol Numerical simulation of optically trapped particles [9289-96]

9289 0J An introductory approach to the concept of spatial coherence [9289-100] 
9289 OK Integrating undergraduate research into the electro-optics and laser engineering technology program at Indiana University of Pennsylvania [9289-12]

9289 OL Interdisciplinary high-school curriculum in electro-optics as a tool to enhance students' interest in optics and electronics [9289-13]

9289 OM Advanced optics in an interdisciplinary graduate program [9289-14]

9289 ON A course on foundations of optical system analysis and design (FOSAD) [9289-15]

928900 Predicting scientific oral presentation scores in a high school photonics science, technology, engineering and mathematics (STEM) program [9289-59]

9289 OP Piloting a fiber optics and electronic theory curriculum with high school students [9289-60]

$92890 \mathrm{OQ}$ Optics in engineering education: stimulating the interest of first-year students [9289-83]

9289 OR Course for undergraduate students: analysis of the retinal image quality of a human eye model [9289-87]

9289 OS Motivational activities based on previous knowledge of students [9289-88]

\section{SESSION $3 \quad$ PROGRAM DEVELOPMENT}

9289 OT The high education of optical engineering in East China [9289-16]

9289 OU The evolution of optics education at the U.S. National Optical Astronomy Observatory [9289-18]

9289 OV Developing intra-curricular photonics educational material for secondary schools in Europe [9289-17]

9289 OW UNESCO active learning approach in optics and photonics leads to significant change in Morocco [9289-19]

9289 0X Graduate studies on optoelectronics in Argentina: an experience [9289-61]

9289 OY ALOP-active learning in optics and photonics: a UNESCO's program spreading in Colombia through the National University [9289-62]

$9289 \mathrm{OZ}$ Contribution from optical course for the educational guidance of engineering careers students [9289-65]

928910 Teaching methodologies to promote creativity in the professional skills related to optics knowledge [9289-72]

$928911 \quad$ NEMO educational kit on micro-optics at the secondary school [9289-76] 
928912 A proposal on teaching methodology: cooperative learning by peer futoring based on the case method [9289-81]

928913 Building an optomechatronics group in a young university in Western Romania [9289-93]

928914 Expansion of student activities in Africa: from south to north [9289-94]

928915 Using concept building in optics to improve student research skills [9289-102]

928916 Towards a research pole in photonics in Western Romania [9289-106]

\section{SESSION 4 CONCEPTUAL UNDERSTANDING ASSESSMENT}

928917 Lighting the way: photonics leaders II (PL2) optics and photonics teacher professional development [9289-20]

928918 Student reactions to problem-based learning in photonics technician education [9289-21]

928919 Light and optics conceptual evaluation findings from first year optometry students [9289-22]

9289 1A Misconceptions about optics: An effect of misleading explanations? [9289-23]

9289 1B Scientific evaluation of an intra-curricular educational kit to foster inquiry-based learning (IBL) [9289-92]

\section{SESSION $5 \quad$ TRAINING AND LABORATORY DEMONSTATIONS}

9289 1C Learning to teach optics through experiments and demonstrations [9289-24]

9289 1D Multicolour LEDs in educational demonstrations of physics and optometry [9289-25]

9289 IE Visualization of light beams in liquid crystal layers for demonstration of basic optical phenomena [9289-26]

9289 IF A laboratory module on radiometry, photometry and colorimetry for an undergraduate optics course [9289-27]

9289 1G Quantum optics laboratories for undergraduates [9289-28]

9289 1H Slit-lamp management in contact lenses laboratory classes: learning upgrade with monitor visualization of webcam video recordings [9289-73]

$928911 \quad$ Teaching optics with the centennial universal lantern [9289-104]

9289 i New frontiers in color management by using modern spectrometers [9289-105]

9289 1K Development of an undergraduate optics laboratory based on the analysis of digital images [9289-107] 
$92891 \mathrm{~L}$ Calculation of reflected and transmifted powers of a metamaterial waveguide structure using MAPLE software [9289-29]

$92891 \mathrm{M}$ Learning about light and optics in on-line general education classes using at-home experimentation [9289-30]

9289 iN Internet based post-graduate course in spectacle lens design [9289-31]

928910 HOLONET: a network for training holography [9289-32]

9289 IP Interdisciplinary education in optics and photonics based on microcontrollers [9289-33]

9289 1Q Advantages and disadvantages of using computers in education and research [9289-34]

9289 IR Learning in the cloud: a new challenge for a global teaching system in optics and photonics [9289-35]

9289 is Web-based interactive educational software introducing semiconductor laser dynamics: Sound of Lasers (SOL) [9289-36]

9289 IT Design, development, testing and validation of a Photonics Virtual Laboratory for the study of LEDs [9289-37]

9289 iU Graphical user interfaces for teaching and research in optical communications [9289-38]

9289 IV Diversity of devices along with diversity of data formats as a new challenge in global teaching and learning system [9289-39]

9289 IW MATLAB GUI (graphical user interface) for the design of GRIN components for optical systems as an educational tool [9289-74]

9289 1X Development of Matlab GUI educational software to assist a laboratory of physical optics [9289-75]

9289 IY Optics in the physics degree at the USC: the use of the Moodle platform [9289-77]

928912 Master on Photonics and Laser Technologies: on-line teaching experience [9289-78]

928920 A teaching resource using the GUIDE environment: simplified model of the eye for secondary school students [9289-84]

928921 Virtual-reality-based educational laboratories in fiber optic engineering [9289-98]

928922 Laws of reflection and Snell's law revisited by video modeling [9289-103] 
928923 DEFI Photonique: a French national training project for optics and photonics industry [9289-40]

928924 Getting Light to Work: photonics up-skilling for industry [9289-41]

928925 MSc degree in color technology for the automotive sector [9289-42]

928926 ANSI laser standards, education (Z136.5), research, development or testing (Z136.8) [9289-43]

928927 Optical inspection methods and their applications in the manufactured industrial sector: knowledge transfer to Panamanian industry [9289-44]

928928 First optical education center in Japan established by cooperation between academia and industry [9289-45]

\section{SESSION 8 HANDS-ON AND OUTREACH}

928929 Workshop on active learning: two examples [9289-46]

9289 2A Student activity: verification on Malus's law of polarization at low cost [9289-47]

9289 2B Shedding the light on spectrophotometry: the SpecUP educational spectrophotometer [9289-48]

9289 2C Photonics Explorer Workshop [9289-49]

9289 2D The LuNa project: experimental didactic modules exploiting portable setups to teach optics in primary and secondary schools [9289-50]

$92892 \mathrm{E} \quad$ Light on the Waves: Science, music, poetry... and light! [9289-51]

9289 2F The Galileoscope project: community-based technology education in Arizona [9289-52]

$92892 \mathrm{G}$ Following the path of light: recovering and manipulating the information about an object [9289-53]

9289 2H The PHOTON explorations: sixteen activities, many uses [9289-54]

$928921 \quad$ Hands-on optics and photonics outreach in Riga [9289-55]

$92892 \mathrm{~J}$ The magic of light! An entertaining optics and photonics awareness program [9289-56]

9289 2K The Hands-On Optics Project: a demonstration of module 3-magnificent magnifications [9289-57]

9289 2L On-light: optical social network [9289-66] 
9289 2M Investigating shadows: a pedagogical intervention project with primary school children [9289-68]

$92892 \mathrm{~N}$ Light: an experiments based learning approach with primary school children [9289-69]

928920 Advanced experiments with an erbium-doped fiber laser [9289-70]

$92892 \mathrm{P} \quad$ Learning optics using a smart-phone [9289-71]

$92892 \mathrm{Q}$ Incorporating active-learning techniques into the photonics-related teaching in the Erasmus Mundus Master in "Color in Informatics and Media Technology" [9289-79]

$92892 \mathrm{R}$ Measuring the image quality of digital-camera sensors by a ping-pong ball [9289-80]

$928925 \quad$ Naked-eye astronomy: optics of the starry night skies [9289-82]

9289 2T The USC-OSA Student Chapter: goals and benefits for the optics community [9289-85]

$92892 \mathrm{U}$ Pick it up with light! An advanced summer program for secondary school students [9289-89]

9289 2V Active learning in optics and photonics: Fraunhofer diffraction [9289-95]

$92892 \mathrm{~W}$ Hands-on physics displays for undergraduates [9289-101]

\section{SESSION 9 WOMEN IN SCIENCE}

9289 2X Women in science: physics and optics [9289-58] 


\section{Authors}

Numbers in the index correspond to the last two digits of the six-digit citation identifier (CID) article numbering system used in Proceedings of SPIE. The first four digits reflect the volume number. Base 36 numbering is employed for the last two digits and indicates the order of articles within the volume. Numbers start with 00, 01, 02, 03, 04, 05, 06, 07, 08, 09, 0A, 0B...0Z, followed by 10-1Z, 20-2Z, etc.

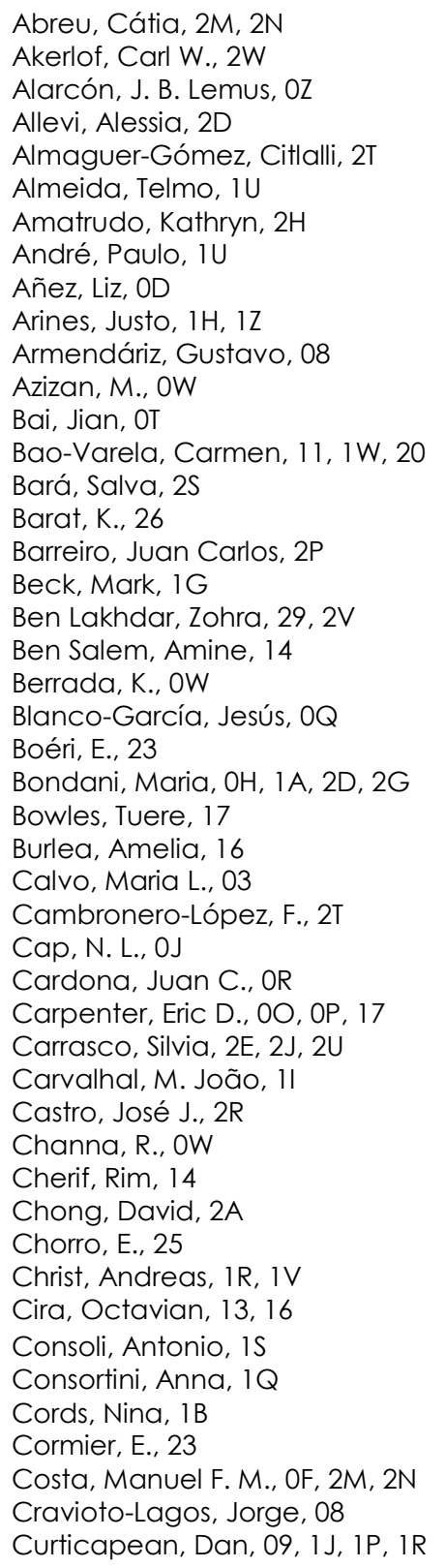

Davies, Ray, 24

Debaes, Nathalie, OV, 1B, 2C

Dederick, Ethan, $1 G$

Delgado-García, Tamara, 2T

del Mar Lázaro, M., 12

del Mar Pérez, Maria, OR

Demian, Dorin, 13, 16

Dhouaidi, Z., 2V

Díaz, Leonardo, OD

Dionísio, Rogério P., 2L

Dokter, Erin F. C., 2F

Donnelly, Judith, 18, 2H

Dreßler, Paul, 1 J, 1P

Dugan, Charles L., 2F

Duma, Virgil-Florin, 13, 16

Durbán, Juan José, 12

Esquivias, Ignacio, is

Euler, Manfred, 1B

Fairchild, Mark D., 07

Farsakoglu, O. Faruk, 02

Favale, Fabrizio, 1A, 2D, 2G

Feisst, Markus, IR

Fernández, Elena, $1 \mathrm{X}$

Fernández, Juan C., OX

Fernandez, Paz, 10

Fernández-Alonso, Mercedes, 1C

Fernández-Oliveras, Alicia, OR, 10

Ferreira, Carlos, OG

Fine, Leonard W., 2F

Fiorucci, M. Paula, 1K

Fischer, Robert, OV, 1B

Florensa, Carlos, 2J

Flores-Arias, M. Teresa, 11, 1Y, 1Z, 2T

Forbes, Andrew, 14

Forbes, Patricia B. C., 2B

Friedman, Jonathan S., OA

Fuentes, Rosa, $1 \mathrm{X}$

Galvez, E. J., 08

García, Celia, 1X

García, J. A., OS

García-Martínez, Pascuala, 0G, 2P

Garcia-Matos, Marta, 2E

Garea, María T., OX

Gargallo, Ana, 1H, 2T

Gero, Aharon, OL

Ghalila, H., 2V

Gheorghiu, Nicolae, 16 
Ghinea, Razvan, OR

Gilabert, E., 25

Gilchrist, Pamela Olivia, 00, OP, 17

Gómez-Robledo, L., OS

Gómez-Varela, A. I., 1W, 20, 2T

González Núñez, Héctor, 2T

González, J. J. Llovera, 0 Z

Gray-Battle, Asia, 00, OP

Grether, Marcela, 08

Grove, Timothy T., 15

Grumel, E., OJ

Gueddana, Amor, 14

Haiss, Ulrich, $1 \mathrm{~J}, 1 \mathrm{P}$

Hanes, Fenna, 18, 2H

Hayes, Dana, 21

Hazra, Lakshminarayanan, ON

Heidt, Alexander M., 14

Hernández-Andrés, Javier, $2 Q$

Hilliard-Clark, Joyce, 17

Horche, Paloma R., is

Huertas, R., OS

Hutiu, Gheorghe, 13, 16

Inal Atik, Ipek, 02

Ionescu, Ana M., OR

Isaurralde, Silvia, OX

Jaafar, Rosly, 2A

Jalie, M., IN

Kadri, Shahrul, 2A

Kaposta, losif, 13

Kocabas, Hikmet, 02

Kozhevnikov, Michael, 21

Kumar, S. Chaitanya, 2J, $2 \mathrm{U}$

Lahmar, Souad, 29, 2V

Lakshminarayanan, Vasudevan, 06, 19, 29

Lancis, Jesús, 1C

Lasso, William, OD

Lesina, Natalija, 2l

Liu, Xiangdong, OT

Liv, Xu, OT

Liu, Yuling, OT

Livshits, Irina, $O B$

López, Ana J., 1K

López-Moreno, Enrique, 08

Magalhães, D. S. F., $0 Z$

Majdi, Y., 2V

Maksimochkin, A. G., $1 \mathrm{E}$

Marcauteanu, Corina, 16

Marques, Manuel B., 11, 20

Marques, Paulo V.S., 20

Martí, Míriam, 2J

Martínez, Guadalupe, OC, IT

Martínez-León, Lluis, 1C

Martinez-Verdu, F., 25

Massa, Nicholas M., 18

Masters, Mark F., 15, 1M

Mathew, Manoj, $2 U$

Melgosa, Manuel, 07
Meyrueis, Patrick, IV

Michinel, Humberto, $1 \mathrm{Z}$

Millspaw, Jacob, 1M

Mínguez-Vega, Gladis, $1 C$

Miranda Díaz, Lázaro J., OE

Miutescu, Eftimie, 16

Mnerie, Corina, 13, 16

Monroy-Ramírez, Freddy Alberto, oY

Monteiro, Marisa, 11

Moreno, Ignacio, 0G, 2P

Muramatsu, M., $\mathrm{OZ}$

Naidoo, Darryl, 14

Naranjo, Francisco L., OC, $1 T$

Nardo, Luca, 2D

Navas, Marianela, OD

Negrutiu, Meda L., 16

Nic Chormaic, S., $O M$

Nieves, Juan Luis, $2 Q$

Nogueira, Rogerio, $1 U$

Nöthling, Johan A., 2B

Noversa, Silvana, 2M, 2N

Oliveras, Maria Luisa, 10

Overiagli, A., OW

Outzourhit, A., OW

Ozolinsh, Maris, ID

Pardo, Pedro J., $1 T$

Paredes, Ángel, 12

Pareja, D. Zottola, $\mathrm{OZ}$

Pascual, Inmaculada, IX

Pasechnik, S. V., $1 \mathrm{E}$

Paulins, Paulis, 1D

Peinado, A., $2 X$

Peña-García, Antonio, 10

Perales, E., 25

Perales, F. J., OS

Pérez, Ángel Luis, OC, $1 T$

Pérez, I. Alfonso, $\mathrm{OZ}$

Perez, Liliana I., OX

Pérez-Ocón, Francisco, 2R

Pino, Abdiel O., 27

Pladellorens, Josep, 27

Podoleanu, Adrian Gh., 16

Polak, Robert D., IF

Pombo, Pedro, 10

Pompea, Stephen M., OU, 2F, 2K

Pons, Amparo, 2P

Pozo, Antonio M., 12, 2Q, 2R

Prasad, Amrita, OV, 1B, 2C

Rabal, H. J., OJ

Raffo, Carlos A., OX

Ramil, Alberto, $1 \mathrm{~K}$

Ramírez-Gómez, Catalina, OY

Rice, Jonny, 21

Robinson, Kathleen, $2 \mathrm{H}$

Rodrigues, M., 22

Rohwer, Erich G., 14

Rolland, Jannick P., 16 
Rominu, Mihai, 16

Rosa, Carla C., 20

Rubiño, Manuel, 2Q, 2R

Salas, Carlos, 12, 2R

Salgueiro, José Ramón, $1 Z$

Salvado-Vara, F., 20

Sanchez, Jorge Rodero, 15

Santos, Emanuel, 10

Shabat, Mohammed M., $1 \mathrm{~L}$

Shmeliova, D. V., $1 \mathrm{E}$

Shoop, Barry L., 04

Shore, K. Alan, 05, 24

Sid-Ahmed, Mohammed O., $1 \mathrm{~L}$

Simeão Carvalho, P., 22

Sinescu, Cosmin, 16

Sparks, Robert T., OU, 2F, 2K

Spigulis, Janis, 2

Suero, María Isabel, OC

Sultana, Razia, 1R, 1V

Tajahuerce-Romera, Enrique, IC

Thapa, Damber, 19

Thienpont, Hugo, OV, 1B

Toledo, R. Serra, $0 Z$

Topala, Florin, 16

Torres, César O., OD

Trivi, M., OJ

Turczynski, Craig, 21

Ubeid, Muin F., $1 \mathrm{~L}$

Urdaneta, Romer, OD

Valencia, Alejandra, $2 U$

Varela, Paulo, 2M, 2N

Vasilyev, Vladimir, OB

Vauderwange, Oliver, 1 J, $1 \mathrm{P}$

Vázquez-Dorrío, Benito, OQ, 12

Velázquez, Victor, 08

Vincitorio, Fabio, $1 \mathrm{~K}$

Viqueira, $V ., 25$

Vlascici, Miomir, 16

Voiculescu, loana, 16

Volpe, Giorgio, 0I, $2 \mathrm{U}$

Volpe, Giovanni, OI, $2 \mathrm{U}$

Walker, Constance E., OU, 2F, 2K

Wang, Gang, 1M

Wang, Xiaoping, OT

Wei, Bor, 2A

Wielage, Heinz-Hermann, 1 J, $1 \mathrm{P}$

Yáñez, Armando, 12

Yatagai, Toyohiko, 28

Yebra, Ana, OR

Yeras, A. Moreno, $\mathrm{OZ}$

Yzuel, M. J., 2X

Zach, Efrat, OL

Zghal, Mourad, 14

Zhou, Andrew F., OK 
Proc. of SPIE Vol. $9289928901-12$

Downloaded From: https://www.spiedigitallibrary.org/conference-proceedings-of-spie on 26 Apr 2023 Terms of Use: https://www.spiedigitallibrary.org/terms-of-use 


\title{
Conference Committee
}

\author{
Conference Chairs
}

Manuel F. P. C. Martins Costa, Universidade do Minho (Portugal)

Mourad Zghal, University of Carthage (Tunisia)

Program Committee

Vasudevan Vengu) Lakshminarayanan, Chairperson, University of Waterloo (Canada)

Alan Shore, Photonics Academy for Wales and Bangor University (United Kingdom)

Anand Krishna Asundi Nanyang Technological University (Singapore) and Optics and Photonics Society of Singapore (Singapore)

Clementina Timus, INFLR (Romania)

Cristiano M. B. Cordeiro, Universidade Estadual de Campinas (Brazil)

Efraín Solarte Rodriguez, Universidad del Valle (Colombia)

Eric Rosas, Centro de Investigaciones en Óptica, A.C. (Mexico) and Red Iberoamericana de Óptica (Mexico)

Guillermo Baldwin, Pontificia Universidad Católica del Perú (Peru)

Helder Crespo, Universidade do Porto (Portugal)

Humberto Michinel, Universidade de Vigo (Spain)

Jesús Blanco, Universidade de Vigo (Spain)

Joaquim Carneiro, Universidade do Minho (Portugal)

José Benito Vazquez-Dorrio, Universidade de Vigo (Spain)

José Figueiredo, Universidade do Algarve (Portugal)

José Luis Paz, Universidad Simón Bolívar (Venezuela)

José Luis Santos, Universidade do Porto (Portugal)

Lúcia Bilro, Instituto de Telecomunicações, Aveiro (Portugal)

Maité Flores-Arias, Universidade de Santiago de Compostela (Spain)

Manuel Joaquim Marques, Universidade do Porto (Portugal)

Marcelo Trivi, Universidad Nacional de Mar del Plata (Argentina)

Maria Luisa Calvo-Padilla, Universidad Complutense de Madrid (Spain)

Maria Sagrario Millan, Universitat Politècnica de Catalunya (Spain)

Marlos Viana, University of Illinois (United States)

Marta Ramos, Universidade do Minho (Portugal)

Maxim Tomilin, University of St. Petersburg (Russian Federation)

Mikiya Muramatsu, Universidad de São Paulo (Brazil)

Orlando Frazão, INESC Porto (Portugal)

Paulo Fiadeiro, Universidade da Beira Interior (Portugal)

Paulo Tavares, Universidade do Porto (Portugal) 
Paulo Simeão, Universidade do Porto (Portugal)

Pedro Andrés, Universitat de València (Spain)

Roger Ferlet, Université Paris Diderot - Paris VII (France)

Rogério Nogueira, Instituto de Telecomunicações, Aveiro (Portugal)

Salvador Bará, Universidade de Santiago de Compostela (Spain)

William T. Rhodes, Florida Atlantic University (United States)

Zuqing Zhu, University of Science and Technology of China (China

International Scientific Committee

Zohra Ben Lakhdar, Chairperson, Faculty of Sciences Tunis STO-TN

(Tunisia)

Abdenbi Bouzid, Université Moulay Ismail Présidence Meknès

(Morocco)

Ahmadou Wagué, Université Cheikh Anta Diop (Senegal)

Ajoy Ghatak, Indian Institute of Technology Delhi (India)

Alan Shore, Photonics Academy for Wales and Bangor University (United Kingdom)

Alex Mazzolini, Swinburne University of Technology (Australia)

Ángela M. Guzmán, CREOL, The College of Optics \& Photonics,

University of Central Floria (United States)

Anna Consortini, Università degli Studi di Firenze (Italy)

Ari Friberg, KTH Royal Institute of Technology (Sweden)

Armando Dias Tavares, Universidade Federal do Rio de Janeiro (Brazil)

Asticio Vargas, Centro de Óptica y Fotónica (Chile)

Barry Shoop, U.S. Military Academy (United States) and Worcester Polytechnic Institute (United States)

Brian J. Thompson, University of Rochester (United States)

Carlos Ferreira, Universitat de València (Spain)

Christiaan Velzel, Nederlandse Philips Bedrijven B.V. (Netherlands)

Daniel Malacara Hernández, Centro de Investigaciones en Óptica, A.C. (Mexico)

David Sokoloff, University of Oregon (United States)

Deb Kane, Macquarie University (Australia)

Elizabeth Simmons, Michigan State University (United States)

Eric Rosas, Centro Nacional de Metrología (Mexico) and Red Iberoamericana de Óptica (Mexico)

Fernando Mendoza, Centro de Investigaciones en Óptica, A.C. (Mexico)

François Flory, École Centrale de Marseille (France)

Gonçalo Figueira, Instituto Superior Técnico (Portugal)

Hector Rabal, Centro de Investigaciones Ópticas (Argentina)

Humberto Michinel, Universidade de Vigo (Spain)

Humbertus Bergman, Stellenbosch University (South Africa)

Imrana Ashraf, Quaid-I-Azam University (Pakistan)

Ivan Culaba, Ateneo de Manila University (Philippines) 
Javier Sanchez Mondragon, Instituto Nacional de Astrofisica, Óptica y Electrónica (Mexico) and Universidad Autonóma del Estado de Morelos (Mexico)

Joaquín Campos Acosta, Instituto de Óptica, CSIC (Spain) and SEDOPTICA (Spain)

Joe Niemela, International Center for Theoretical Physics (Italy)

Joel Maquiling, Ateneo de Manila University (Philippines)

John Love, Australian National University (Australia)

José Manuel de Nunes Vicente Rebordão, Universidade Nova de Lisboa (Portugal)

Katarina Svanberg, University of Lund (Sweden)

Kathleen Robinson, SPIE

Khalid Berrada, Cadi Ayyad University, Marrakech (Morocco)

Liu Xu, Zhejiang University (China)

Luc Owonou, Doualy University (Cameroon)

Lucilia Cescato, Universidade Estadual de Campinas (Brazil)

Luis Miguel Bernardo, Universidade do Porto (Portugal)

M. J. Soileau, CREOL, The College of Optics \& Photonics, University of Central Floria (United States)

Mama Mnsangou, Université de Ngaoundéré Cameroun, Université de Maroua (Cameroon)

Manuel Filipe Costa, Universidade do Minho (Portugal)

Manuel Melgosa Latorre Universidad de Granada (Spain)

Marc Nantel, University of Toronto (Canada)

María Josefa Yzuel Giménez, Universitat Autònoma de Barcelona (Spain)

Maria Luisa Calvo-Padilla, Universidad Complutense de Madrid Spain)

Maria Sagrario Millan, Universitat Politècnica de Catalunya (Spain)

Mauricio Pietrocola, Universidad de São Paulo (Brazil)

Maxim Tomilin, University of St. Petersburg (Russian Federation)

Mikhail Vasilevski, Universidade do Minho (Portugal)

Mohammed M. Shabat, Islamic University of Gaza (Palestine)

Mourad Zghal, University of Carthage (Tunisia)

Mustafa Erol, Bozok University (Turkey)

Pablo Artal, Universidad de Murcia (Spain)

Paul Buah-Bassuah, University of Cape Coast (Ghana)

Pierre Chavel, Institut d'Optique (France)

Radu Chisleag, University Politehnica of Bucharest (Romania)

Ramón Rodríguez-Vera, Centro de Investigaciones en Óptica, A.C. (Mexico)

Roberta Ramponi, Politecnico di Milano (Italy)

Rogério Nogueira, Instituto de Telecomunicações, Aveiro (Portugal)

Tahani Mohamed Shatir, University of Khartoum (Sudan)

Tuan-Kay Lim, Nanyang Technological University (Singapore)

Vasudevan (Vengu) Lakshminarayanan, University of Waterloo (Canada) 
Zaia Derrar Kaddour, Université Science and Technology Haouari Boumediene-Alger (Algeria)

Zhu Bingkun, University of California, Davis (United States)

Proc. of SPIE Vol. $9289928901-16$

Downloaded From: https://www.spiedigitallibrary.org/conference-proceedings-of-spie on 26 Apr 2023 Terms of Use: https://www.spiedigitallibrary.org/terms-of-use 